\title{
Cultura histórica e livro didático ideal: algumas contribuições de categorias rüsenianas para um ensino de História à brasileira
}

\author{
Itamar Freitas Oliveira* \\ Margarida Maria Dias de Oliveira*
}

\section{Resumo}

Este artigo apresenta e problematiza a cultura histórica e o livro didático a partir de algumas obras do historiador Jörn Rüsen, sobretudo o texto O livro didático ideal (2010). Com este, deseja-se contribuir para a discussão em relação ao emprego das referidas categorias, disponibilizadas aos pesquisadores brasileiros do campo do ensino de História, principalmente, em termos de produção e avaliação de livros didáticos, além das demais práticas do ensino de História em sala de aula. Para cumprir tal objetivo, o texto (1) menciona algumas iniciativas de produção de princípios e critérios para a aferição da qualidade do livro didático de História; (2) aborda as ideias de Homem e cultura em Jörn Rüsen e de livro didático de História; (3) relaciona as prescrições do livro didático ideal de Jörn Rüsen e a apresentação de uma didática para a História; e (4) anuncia reflexões referente à possibilidade de uma didática rüseniana à brasileira.

Palavras-chave: Cultura histórica. Livro didático. Ensino de História.

\section{Introdução}

O livro didático ideal está nas mentes de cada profissional de História. Essa é uma assertiva presente no senso comum dos historiadores. Na condição de unanimidade, esse pensamento explicita um problema para os pesquisadores: aceitar a impossibilidade de critérios relativamente consensuais acerca da qualidade do livro didático de História ou investigar a fundo os autores que se dispõem a sugerir critérios que indiquem as características que melhor contribuam para o cumprimento das finalidades do ensino de História.

Recebido: 25/04/2014 - Aprovado: 19/06/2014

http://dx.doi.org/10.5335/rep.v21i2.4298

Doutor em Educação, Professor do Departamento de Educação e do Programa de Pós-Graduação em História da Universidade Federal de Sergipe. E-mail: itamarfo@gmail.com.

* Doutora em História e Professora do Programa de Pós-Graduação em História e do curso de Licenciatura em História da Universidade Federal do Rio Grande do Norte (PPGH/UFRN). E-mail: margaridahistoria@yahoo.com.br. 
Várias instituições têm atuado nesse sentido, nas últimas duas décadas, no Brasil e no exterior. Para não nos alongarmos em demasia, citemos apenas o próprio Ministério da Educação brasileiro, por meio dos agentes que produzem os editais do Programa Nacional do Livro Didático (PNLD) (FILGUEIRAS, 2011; OLIVEIRA; FREITAS, 2013), do Ministério da Educação Nacional francês, do seu corpo de inspetores nacionais (BORNE, 1998), do Ministério de Educación do Chile, mediante o suporte de consultores internacionais, durante o Primer Seminario Internacional de Textos Escolares (MEC, 2006) e entidades civis estadunidenses como o American Textbook Council (SEWALL, 2000).

Pesquisadores também inventariam princípios, critérios "grilles", visando à sofisticação desse poderoso instrumento didático. Dos mais ativos, nesse sentido, são Rafael Valls (2008), Joaquín Prats (2012) e Jörn Rüsen (2010).

No Brasil, Rüsen tem sido o mais citado, desde a publicação do artigo intitulado "El libro didático ideal" (2010), traduzido para o português (2012). Um dos autores que mais próximo chegaram do restrito emprego das diretrizes rüsenianas foi Daniel Hortêncio de Medeiros (2005). As conclusões apresentadas, na sua tese de doutoramento, anunciam o alinhamento aos critérios defendidos por Rüsen, como exemplificado no excerto que se segue:

Como procurei definir no capítulo dois, as condições materiais de produção dos manuais didáticos de História submetem sua feitura não às possibilidades de produção de consciência histórica, mas ao movimento de aquisição de bens, voltando sua racionalidade para a consecução deste objetivo.

Tais procedimentos implicam uma interferência determinante na seleção dos conteúdos e na disposição das narrativas oferecidas aos jovens alunos e alunas. Assim, enquanto para Rüsem (1997a, p. 35), um bom livro de texto é um livro capaz de ensinar para a percepção histórica, de oferecer a possibilidade de realizar interpretações históricas corretas e estimular a orientação para a vida presente, relacionando-as com as perspectivas de futuro, para a editora que produziu o manual didático da pesquisa, o melhor material didático é o que facilita o aprendizado do aluno/a. Este tem maior aceitação no mercado, pois é bom para o professor (MEDEIROS, 2005, p. 160, grifos do autor).

Fora do Brasil, exemplo significativo do uso das diretrizes do teórico alemão está nos trabalhos de Rafael Valls (2008) que não somente incorpora alguns dos seus métodos em uma proposta integradora de critérios de avaliação e julgamento de livros didáticos espanhóis, como considera ser a de Rüsen

[...] la más estritcta y genuinamente didáctica, em su planteamento básico [...]. Para este autor, la enseñanza de la historia debe de sumisnistrar a los alumnos lo que el define como uma competência narrativa, esto es, la capacidad de entender las relaciones que el presente tiene o estabelece com el pasado y com las perspectivas de futuro (DHIGECS, 2008, p. 69). 
Aqui, trilhamos os caminhos desses autores, mas sem a pretensão de julgar o melhor conjunto de critérios ou a necessidade de aplicá-lo(s) em sua totalidade. Nosso objetivo é apresentar, resumida e esquematicamente, alguns dos sentidos de cultura histórica e de livro didático, disseminados por Jörn Rüsen, demonstrando possibilidades de articulação entre tais conceitos, bem como anunciar alternativas de emprego da referida relação (cultura histórica/livro didático) na pesquisa histórica dos livros didáticos de História, a partir de uma matriz. Em seguida, apresentamos algumas reflexões em relação aos elementos originais e os indícios e suportes da vulgata pedagógica ocidental, presentes na escrita de Jörn Rüsen, sobretudo em seu texto prescritivo acerca do livro didático de História.

$\mathrm{Na}$ exposição, entretanto, não nos limitaremos à discussão dos sentidos de cultura histórica e de livro didático. Nossa estratégia é inverter a abordagem tradicional dos estudos históricos sobre teoria e iniciar com perguntas "metafísicas", a exemplo dos sentidos de homem e de vida na ontologia rüseniana.

\section{0 homem e a cultura}

O que é o homem de Jörn Rüsen? Estevão de Rezende Martins apresenta o teórico como adepto do humanismo intercultural, isto é, filiado a uma perspectiva que busca unicidade na diversidade humana, que persegue e sugere "uma espécie de mínimo denominador comum a todo e qualquer agente racional humano" (MARTINS, 2010, p. 8).

O humanismo fundante, que Rüsen adota e expõe, constitui-se em uma espécie de mínimo denominador comum a todo e qualquer agente racional humano, pouco importa onde, quando ou como. Para além do laivo metafísico presente nessa visão, seu substrato é a concepção geralmente aceita dos direitos do homem e do cidadão, em suas diversas expressões desde a declaração da Virgínia, em 1776, até as que vieram a ser concretamente debatidas e adotadas no espaço público internacional a partir de 1948. A historicidade empírica da realidade das sociedades e das culturas, que envolve a cada um de nós como indivíduo pensante e agente, é, por conseguinte, o ponto de partida para encontrar, em sua diversidade, o que nos faz iguais, livres e solidários. Ou o que nos deveria fazer iguais, livres e solidários (MARTINS, 2010, p. 8).

Além das palavras de Martins, não pensamos ser exagerado afirmar: Rüsen é um pensador que define e (definindo, obviamente) inventa uma natureza humana. Qual é a natureza do homem? O que o singulariza perante os demais seres vivos? O que (do homem) o faz homem? À primeira vista, Rüsen não se distancia dos vários pensadores/epistemólogos que tentaram resolver essas questões sem a intervenção divina. Herbert Spencer, Émile Durkheim, John Dewey, Jean Piaget - somente para citar alguns dos mais conhecidos filósofos que pensaram a aprendi- 
zagem histórica - conceberam o homem como dotado das faculdades ou poderes de agir, pensar e sentir. Com as devidas licenças ao anacronismo, portanto, podemos afirmar que todos esses pensaram o homem como produtor e produto da cultura, a qual é aqui entendida em oposição à natureza. Assim, também procede Rüsen, quando caracteriza o homem como um ser dotado de "sentimento, vontade e intelecto" (RÜSEN, 1994, p. 21). Seria, então, a cultura o processo e o resultado (ações e artefatos) do sentir, do agir e do conhecer.

Mas, por que afirmamos que seria em lugar de é? Ora, a proximidade com os citados pensadores do século XIX e da primeira metade do XX, encerra-se no esquema triádico de conceber o homem. Isso porque Rüsen introduz a ideia de tempo (agir/ sofrer/orientar-se - produzir sentido no tempo) e enuncia (pensamos) aquilo que Estevão Martins classificou de mínimo denominador comum e que o próprio autor alemão nomeia de universais antropológicos. Como temos muitas dúvidas relacionadas a alguns sentidos expressos na sua teoria, repartiremos esses universais antropológicos em dois elementos: (1) "o modo histórico de viver do homem" (RÜSEN, 1994, p. 5), necessariamente, agindo e orientando-se no tempo; e (2) "a natureza espiritual-mental do homem" (RÜSEN, 1994, p. 5), isto é, lugar em que operam a percepção, a interpretação e a representação do passado (RÜSEN, 2006, p. 118). ${ }^{1}$

\section{Cultura histórica}

Pelo exposto, podemos perceber que o termo cultura é empregado em dois sentidos. Um conceito amplo que abrange todo o agir/sofrer significativo do homem - o processo e o resultado do sentir, agir e do conhecer enfeixados nas rubricas tão conhecidas por nós, como a economia, a arte, a política, a sociedade e a cultura ou, ainda, tudo o que o homem faz. Por outro lado, cultura é também um conceito restrito a apenas uma parte da práxis vital, a que abrange as operações da consciência.

Ambos os termos constituem modos (respectivamente, de viver e de conhecer) universais de produção de sentido (orientação no tempo), cabendo-lhes, pois, o conceito de cultura histórica. Assim, os sentidos de cultura histórica são intercambiáveis: a consciência (parte da vida prática) determina a (totalidade da) vida prática e esta determina a consciência (RÜSEN, 1994, p. 6).

Não obstante a dicotomia, Rüsen parece optar pelo sentido estrito quando afirma que:

La cultura histórica se refiere por tanto a una manera particular de abordar interpretativamente el tiempo, precisamente aquella que resulta em algo como "historia" en cuanto contenido de la experiencia, producto de la interpretación, medida de orientación y determinación de la finalidad (RÜSEN, 1994, p. 6, grifos nossos). 
De qué particularidad se trata? Se puede poner de relieve em dos pasos argumentales. Em primer lugar se trata de una aproximación interpretativa del tiempo que se concreta em el modo de la rememoración histórica (RÜSEN, 1994, p. 6, grifos do autor).

La segunda particularidad de la consciência histórica, aparte de la forma temporal de la memória o recuerdo histórico, se refiere al modo de ser de lo memorado [...]. Em realidad existe uma operación así: es la de contar historias (p. 8-9).

Repetindo e concluindo, para Rüsen (e limitadas a tais fontes), a cultura histórica é um conceito que tem a função de nomear esse modo particular (rememorado e narrado) de produzir sentido (orientar-se no tempo) e que está presente (é observável) nas atividades da pesquisa histórica, no ensino de História, nas exposições museais e na mídia, dentre outras dimensões da vida prática (cultura histórica em sentido amplo).

Apesar das escolhas de Rüsen, é importante mantermos na memória os dois sentidos do vocábulo ou as duas dimensões da cultura histórica (consciência e toda a vida prática), caso pretendamos articular cultura histórica e livro didático.

Passemos agora ao segundo termo deste artigo.

\section{Livro didático de História}

Coerente com o valor atribuído ao ensino de História (enraizado na matriz disciplinar - objeto da sua teoria da História), o livro didático é visto como instrumento fundamental para a vida escolar, já que atua, diretamente, na construção do sentido (orientação no tempo).

Rüsen afirma que "antigamente, o livro didático de História era um dos canais mais importantes para levar os resultados da investigação histórica até a cultura histórica de sua sociedade" (RÜSEN, 2010, p. 110). Hoje, a "verdadeira finalidade" de um livro didático de História é "tornar possível, impulsionar e favorecer a aprendizagem da História" (p. 112). Esse chega a afirmar que o "livro de História é o guia mais importante da aula de História" (p. 115).

Pensamos que nesse conjunto de citações estão explícitadas, ao menos, duas relações fundamentais entre os conceitos de cultura histórica e de livro didático. A primeira é a ideia de livro didático como (resultado da) cultura histórica, ou seja, um artefato que materializa (encarna) esse modo particular de produção de sentido (rememoração/narração).

A segunda relação é a ideia de livro didático como meio/processo/instrumento da cultura histórica, cuja finalidade é potencializar as capacidades mentais, ou seja, o livro viabiliza a aquisição e o desenvolvimento das capacidades de perceber, interpretar, orientar e construir a identidade do aluno. 
Seguindo esse último sentido - o livro didático como dinamizador das operações mentais -, somos induzidos a pensar que grande parte dos estudos dedicados a cada um dos ciclos de vida do artefato em discussão (a produção, avaliação e usos do livro didático) deverá ser submetido a uma matriz que inclui como variáveis as já anunciadas "operações" e as "dimensões" fundamentais da mente humana. Dizendo de outro modo, a produção, a avaliação, o uso e os estudos sincrônicos e diacrônicos da produção, da avaliação e dos usos do livro didático de História têm que considerar como conceitos (que geram problemas e prescrições) as operações de experimentar, interpretar e orientar, como também as dimensões estéticas, políticas e cognitivas da mente humana.

Essas proposições, Rüsen as sugere em vários momentos da sua teoria da História (2001, 2007a, 2006b), e nos artigos "O que é a cultura histórica? Reflexões sobre uma nova maneira de abordar a História" (1994) e "O livro didático ideal" (2012). Em especial, destacamos a seguinte passagem:

La diferenciación entre arte, política y ciencia como três âmbitos de la cultura histórica [...] tiene también una base antropológica, pues se puede basar sin problemas en los tres modos fundamentales de la mente humana, en el sentimiento, la voluntad y el intelecto.

[...] las tres dimensiones de la cultura histórica se compenetran mutuamente, y solamente en esa compenetración la conciencia histórica realiza su acción cultural característica, la rememoración histórica.

Así por ejemplo, no se puede pensar ningún texto historiográfico del âmbito de la ciência histórica que no muestre, al lado de las características peculiares de garantia de validez metodológica del conocimiento histórico, princípios de forma estéticos e influencias e intenciones políticas. Puede ser que eso no sea siempre el caso inmediata y directamente, pero seguramente indirectamente y de tal manera, que el texto perderia su sentido, es decir su comprensibilidad, se se prescindiera, en um experimento mental, de los factores de configuración estéticos y políticos. Tampoco hay manifestaciones políticas o estéticas puras de la memória histórica (RÜSEN, 1994, p. 21-22).

Vejamos agora, em formato de matriz, como podem ser representadas as prescrições de Rüsen, que levam em consideração as operações da consciência (cultura histórica) e as dimensões da mente humana (cultura histórica). O exemplo, a seguir, refere-se apenas à produção de livros didáticos. Mas pode, perfeitamente, ser aplicado à avaliação e ao uso em sala de aula. A matriz também oferece questões, hipóteses e conceitos (perspectivas de orientação) à pesquisa histórica com livros didáticos. 
Quadro 1 - O livro didático racional

\begin{tabular}{|c|c|c|c|}
\hline & Sentimento/Estética (beleza) & Vontade/Política (poder) & Intelecto/Ciência (verdade) \\
\hline 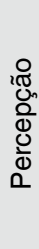 & $\begin{array}{l}\text { - [Provocar] fascinação e } \\
\text { estranhamento (percepção } \\
\text { da diferença). } \\
\text { - [vivenciar] a pluriperspec- } \\
\text { tividade da experiência e da } \\
\text { recepção dos afetados pelos } \\
\text { acontecimentos. }\end{array}$ & & $\begin{array}{l}\text { - [vivenciar] a pluriperspec- } \\
\text { tividade da experiência e } \\
\text { da recepção dos afetados } \\
\text { pelos acontecimentos. }\end{array}$ \\
\hline 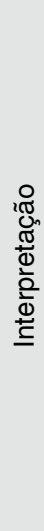 & $\begin{array}{l}\text { [Ser dotado de] força de } \\
\text { convicção na exposição. }\end{array}$ & $\begin{array}{l}\text { - [Ser dotado de] força de con- } \\
\text { vicção na exposição. } \\
\text { - [diferenciar] juízo dos fatos, } \\
\text { hipótese e juízo de valores. }\end{array}$ & $\begin{array}{l}\text { - Correção factual, coerência } \\
\text { interpretativa (em relação } \\
\text { à historiografia erudita), } \\
\text { respeito à ABNT. } \\
\text { - [Possibilitar] problemas, } \\
\text { hipóteses, análise de fon- } \\
\text { tes, interpretação a partir } \\
\text { de teorias. } \\
\text { - } \text { [Informar] sobre a reescrita } \\
\text { da história e a pluriperspec- } \\
\text { tividade dos historiadores } \\
\text { - [Ser dotado de] força de } \\
\text { convicção na exposição. } \\
\text { - [diferenciar] juízo dos fatos, } \\
\text { hipótese e juízo de valores. }\end{array}$ \\
\hline 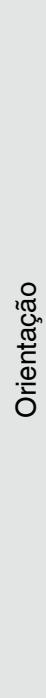 & $\begin{array}{l}\text { Utilizar a referência ao } \\
\text { presente como instrumento } \\
\text { para ilustrar a singularidade } \\
\text { do passado. }\end{array}$ & $\begin{array}{l}\text { - Não somente combater o etno- } \\
\text { centrismo (eurocentrismo, por } \\
\text { exemplo), mas também usar os } \\
\text { conteúdos substantivos para } \\
\text { construir a identidade do aluno } \\
\text { na relação nós/outros. } \\
\text { - [estimular os alunos] para que } \\
\text { emitam juízos de valor sobre } \\
\text { os acontecimentos, argu- } \\
\text { mentando a partir do conceito } \\
\text { que tinham de si mesmos os } \\
\text { afetados pelos acontecimentos } \\
\text { do passado. } \\
\text { - Utilizar a referência ao presente } \\
\text { como instrumento para ilustrar } \\
\text { a singularidade do passado, } \\
\text { perceber as distâncias entre } \\
\text { passado e presente e, com } \\
\text { isso, vislumbrar uma perspec- } \\
\text { tiva futura para o presente. }\end{array}$ & \\
\hline
\end{tabular}

Fonte: construído a partir de Rüsen (1994, p. 21-22; 2010, p. 109-127). 


\section{0 livro didático ideal de Jörn Rüsen e a representação de uma didática para a História}

Quem se predispuser a examinar a obra do filósofo Jörn Rüsen, publicada em português, em busca de sua ideia de ensino de História, por certo encontrará algumas dificuldades, tais como, o caráter abstrato da discussão; a ausência de comentadores dedicados à propedêutica; o sutil distanciamento entre os conceitos, a exemplo de cultura, e de cultura histórica, formação, aprendizado e consciência histórica, etc. Uma dessas, entretanto, não está na própria obra, mas na expectativa que criamos em relação ao que essa pode oferecer.

Certamente há vários trechos da sua teoria da História dedicados à definição, estrutura, forma e função de uma didática da História. O problema é que a didática da História, criticada e prescrita por Rüsen, é um campo de investigação, obviamente, alemão. Esse até esboça algumas definições bastante familiares aos brasileiros, como neste exemplo: a didática histórica é uma disciplina responsável pela formulação da "competência específica para a sala de aula" (RÜSEN, 2007, p. 90). ${ }^{2}$

Mas, quando se debruça objetivamente no tema, informa que o objeto da didática da História é a consciência histórica. $\mathrm{E}$, ainda, que "a didática da História se volta para aqueles processos mentais ou atividades da consciência [...] que geralmente encontram-se por trás dos conteúdos e que habitualmente ficam velados ao aprendiz" (SCHÖRKEN, 1972, p. 84 apud RÜSEN, 2010, p. 42, grifos de SCHÖRKEN). Dito de um modo bem brasileiro, a didática da História de Rüsen ganha o sentido de uma espécie de "psicologia do desenvolvimento", que se preocupa, primordialmente, com a natureza mental dos humanos e não, como esperaríamos no início da leitura, com o anúncio de estratégias para ensinar e aprender História em sala de aula.

E agora? Como minimizar essa quebra de expectativas? Esbocemos a nossa alternativa. No texto que prescreve o livro de História didaticamente correto, nosso filósofo tece considerações gerais acerca da utilidade [do livro didático] para 0 ensino prático. E é, exatamente, nessa exposição despretensiosa - em relação às funções que o livro didático deve cumprir, para além da sua contribuição como canal dos resultados da pesquisa histórica ou do impulso à aprendizagem histórica - que podemos encontrar, de forma clara, algumas respostas relacionadas aos problemas-chave de uma didática da História à brasileira (ou da teoria do currículo à americana).

Dito diferentemente aí nesse fragmento produzido a partir da vulgata sobre a didática da História na Alemanha, podemos colher as ideias de finalidades, seleção e progressão de conteúdos, aprendizagem, estratégias de ensino e avaliação. 


\section{Didática rüseniana à brasileira}

Comecemos com as finalidades. Já sabemos que a História, como disciplina escolar, tem a função de desenvolver as competências de percepção, de interpretação e de orientação, contribuindo para a formação da consciência histórica. Mas, no fragmento em questão, Rüsen indica a necessidade de os alunos terem acesso aos objetivos, às intenções didáticas, ao conteúdo e aos conceitos metodológicos de ensino de forma clara.

A respeito da escolha desses conteúdos, Rüsen afirma que há que expressar uma relação com as experiências e expectativas dos alunos. Em outras palavras, os materiais apresentados aos alunos (documentação, narrativas) e as atividades a esses destinadas têm que ser significativas. É o interesse presente e futuro do aluno quem comanda a seleção do material. O autor, no entanto, ressalva que há que contar também com alguma matéria que contemple as necessidades de orientação no conjunto da sociedade. Embora tais matérias sejam dispostas de forma fragmentada nos conteúdos, a seleção desses, repetimos, deve resumir-se aos interesses individuais dos alunos.

Em se tratando da aprendizagem, Rüsen a discute em todos os volumes da sua teoria. Mas, nesse trecho, desce ao chão da escola quando defende a necessidade de traduzir a matéria às peculiaridades cognitivas dos alunos. É preciso distribuí-la "de acordo com a capacidade de compreensão" (RÜSEN, 2010, p. 116).

Além de pôr o aluno no centro da relação ensino-aprendizado, o teórico também preocupa-se em tornar o processo mais prazeroso. Aí alerta aos profissionais: não há que fazer malabarismos. "A experiência histórica tem um potencial próprio de encantamento que se pode aproveitar com oportunidade de aprendizagem" (RÜSEN, 2010, p. 117). Dizendo de outra forma, a matéria veiculada nas aulas de História é, em si mesma, um reforçador natural. Não é necessário muito esforço para fazer com que os alunos estudem História confortavelmente.

Por fim, a estratégia de ensino. É fundamental estabelecer uma boa relação com o aluno. A ação é simples. Deve o mestre dirigir-se a esse explicitamente. Não esqueçamos que Rüsen está a tratar de livro didático. Mas, pensemos na sala de aula e veremos que o conselho torna-se adequado, apesar da indiferença de muitos mestres com os seus pupilos. A honestidade e a clareza na exposição dos temas e no anúncio da perspectiva teórica de interpretação e a referência direta (estou falando com você) são valores e estratégias que podem convencer o aluno de que ele é realmente o sujeito da aprendizagem, que o professor não está fingindo e, ainda, de que o processo de didatização não significa idiotização. Em termos bem brasileiros, Rüsen propõe uma relação dialógica com o aluno. 
Evidentemente, Rüsen não é um teórico da didática histórica, de metodologia, do ensino, da pedagogia histórica, etc. Em recente encontro com a professora Maria Auxiliadora Schmidt (UFPR), esse fez questão de ressaltar que não pesquisa o ensino de História. "Quem quiser se inteirar desse assunto deve procurar o Borries"3 - referia-se a Bodo Von Borries, que há mais de 35 anos dedica-se à pesquisa referente ao ensino de História na Alemanha.

Apesar de Rüsen não ser um especialista na área e de encontrarmos dificuldades para compreender a sua teoria da História, é possível observar os princípios de uma didática da História que corresponda aos interesses de professores brasileiros, acostumados ao esquema quádruplo: finalidades da disciplina, seleção e progressão dos conteúdos, aprendizagem e ensino. Basta não buscarmos o que costumamos encontrar nos manuais brasileiros de metodologia. Mesmo em texto no qual o filósofo não se propôs, objetivamente, a fornecer diretrizes de ensino-aprendizagem, podemos identificar uma vulgata didática (alemã?), se não elaborada, ao menos, legitimada por Rüsen.

E mais: podemos até situar as suas considerações no curso das correntes pedagógicas circulantes na Europa, Estados Unidos e Brasil no século XX. Com todas as licenças pedagógicas que possamos conseguir, arriscamos dizer que Rüsen, como qualquer teórico sensato, nesse texto, utiliza-se de uma vulgata que inclui as diretrizes de formatação dos objetivos educacionais de Ralph Tyller, a tradução dos objetivos às peculiaridades cognitivas do aluno de Jerome Bruner, a seleção de conteúdos significativos de David Ausubel, a inclusão de conteúdos relativos à satisfação de necessidades sociais de John Dewey e demais progressistas, a ideia de reforço natural de Burrus Skinner e o dialogismo de Lev S. Vigotsky e de Paulo Freire. Em uma palavra, como todo sensato, esse é um eclético. De dogmático, apenas a sua ideia de mente humana fundada nas operações da consciência histórica.

\section{Conclusão}

Neste texto, tentamos convencê-los da existência de uma implicação entre o conceito de cultura histórica como modo particular de produção de sentido e o conceito de livro didático como instrumento potencializador das operações mentais de produção de sentido. ${ }^{4}$ Em outras palavras, se a cultura histórica considera as operações de experimentar, interpretar e orientar e as dimensões estéticas, políticas e cognitivas, os produtores, avaliadores, professores e estudiosos do livro didático de História também devem considerá-las em seus respectivos ofícios.

Com as orientações da teoria da História, o que prescreve Rüsen não é apenas a produção do "livro didático ideal", como denota o título do seu artigo. Esse propõe as diretrizes para o mercado editorial, para as políticas públicas de avaliação e 
ensino de História, um programa de pesquisa histórica e, ainda, os elementos para uma didática à brasileira, se compreendermos que parte do pensamento rüseniano estrutura-se sob a vulgata pedagógica ocidental, produzida ao longo do século XX. Com a ideia de homem e o seu correspondente viabilizador - a consciência histórico-genética -, esse também oferece uma possibilidade de construir um mundo, digamos, menos etnocêntrico.

As indagações que nos vêm à mente, pensando no caso brasileiro, são as seguintes: como conciliar a pluralidade teórica levada à exaustão nos programas de pós-graduação (referente a homem, a História, etc.) com a proposta intercultural de Rüsen? Como adequar a autonomia freireana (vulgata na pedagogia brasileira) com limitada tríade de operações (capacidades) mentais anunciadas por Rüsen? Como garantir a universalização da qualidade (racional - Rüsen) do livro didático com a diversidade de realidades, em termos de horizontes de expectativas e de recursos sociobiológicos (assimetria), da população estudantil brasileira?

Não temos respostas. Entretanto, as questões dão-nos a certeza de que a teoria de Rüsen, como qualquer teoria, enriquece o debate a respeito das finalidades e dos meios da disciplina escolar História, mas não deve, isoladamente, ser elevada à categoria de novo documento oficial do ensino de História no Brasil.

\section{Historical culture and "ideal textbook": some contributions of rüsenianas categories to a "brazilian history teaching"}

\section{Abstract}

This essays presents and discusses the terms "historical culture" and "textbook" from some books from the historian Jörn Rüsen, especially the text "The ideal textbook" (2010). With this, we proposed contribute to the discussion about the use of these categories, available for Brazilians in the field of research teaching of history, especially in terms of production and evaluation of textbooks, besides other practices of teaching history in the classroom. To accomplish this objective, this essay (1) mentions some productions initiatives from principles and criteria for measuring the quality of history textbook, (2) deliberating the ideas of "Man and culture" (3) relates the requirements on the ideal textbook Jörn Rüsen and the presentation of didactic to history (4) announce reflections about the possibility of a "Rüseniana Brazilian Teaching".

Keywords: Historical culture. Textbook. Teaching of History.

\section{Notas}

1 No texto de 1994, essas operações são descritas como percepção, interpretação, orientação e estabelecimento de uma finalidade e percepção, interpretação e orientação (RÜSEN, 1994, p. 6, 20).

2 Diferenciando-a da teoria da História, que é a “didática da ciência da história", isto é, a disciplina responsável pela formação da competência profissional (pesquisa histórica e historiografia). 
3 Informação fornecida durante a palestra proferida no III Seminário de História e Cultura Histórica, promovido pelo Programa de Pós-Graduação em História da Universidade Federal da Paraíba, em João Pessoa, em 28 de setembro 2011.

4 "Relação estabelecida entre dois conceitos ou proposições, de tal forma que a afirmação da verdade de um deles conduz à inferência necessária da veracidade do outro (p. ex., a necessidade da morte implica a inexorabilidade do sofrimento humano)" (HOUAISS, sd).

\section{Referências}

BORNE, Dominique. Le manuel scolaire. [Paris]: inspection génerale de l'Éducation Nationale/ Ministère de l'Éducation Nationale, de la recherche et de la technologie, jun. 1998.

CHILE. MINISTERIO DE EDUCACIÓN. Primer seminario internacional de textos escolares. Santiago de Chile: Unidad de Currículum y Avaluación/Ministerio de Educación de Chile, 2006.

FILGUEIRAS, Juliana Miranda. Os processos de avaliação de livros didáticos no Brasil (19381984). 2011. Tese (Doutorado em Educação) - Programa de Pós-Graduação em Educação História, Política, Sociedade, Pontifícia Universidade Católica de São Paulo, São Paulo, 2011.

MARTINS, Estevão de Rezende. Historicidade e consciência histórica. In: RÜSEN, Jörn. Jörn Rüsen e o ensino de história. Curitiba: Ed. da UFPR, 2010. p. 7-10.

OLIVEIRA, Margarida Maria Dias de; FREITAS, Itamar. Historiografia didática e prescrições estatais sobre conteúdos históricos em nível nacional (1931-2012). Territórios e Fronteiras (Online). Cuiabá, v. 6, n. 3, dez. 2013. Disponível em: <http://www.ppghis.com/territorios\&fronteiras/index. php/v03n02/article/view/242>. Acesso em: 1 fev. 2014.

PRATS, Joaquín. IBER Didáctica de las Ciencias Sociales, Geografía e Historia. n. 70 Enero, Febrero, Marzo 2012. Disponível em: <http://www.ub.edu/histodidactica/index.php?option=com_ content\&view=article\&id=146:criterios-para-la-eleccion-del-libro-de-texto-de-historia\&catid= 9:didactica-de-la-historia\&Itemid=104>. Acesso em: 29 abr. 2013.

RÜSEN, Jörn. História viva: teoria da história - formas e funções do conhecimento histórico. Brasília: Ed. da UnB, 2007.

Historiografia comparativa intercultural. In: MALERBA, Jurandir (Org.). A história escrita: teoria e história da historiografia. São Paulo: Contexto, 2006. p. 115-157.

. Jörn Rüsen e o ensino de história. Curitiba: Ed. da UFPR, 2010.

. O livro didático ideal. In: MARTINS, Estêvão; SCHMIDT, Maria Auxiliadora (Orgs.). Jörn Rüsen e o ensino de história. Curitiba: Ed. da UFPR, 2010. p. 109-127.

SEWALL, Gilbert T. History textooks at the we centrury. Sn: American Textbook Council, 2000.

VALLS, Rafael. Los textos escolares de historia: una propuesta de análisis y valoración. In: PRATS, J.; ALBERT, M. Ells llibres de text i l'ensenyament de la Història. Record d'en Jordi Bruguera Gómez. Grup DHIGECS. Barcelona: Universitat de Barcelona, 2008. 